Article

\title{
Bioactivity-Guided Screening of Wound-Healing Active Constituents from American Cockroach (Periplaneta americana)
}

\author{
Juan-Juan Zhu ${ }^{1}$, Shun Yao ${ }^{2}$, Xin Guo ${ }^{1}$, Bi-Song Yue ${ }^{1}$, Xiu-Ying Ma ${ }^{3}$ and Jing $\mathrm{Li}^{1 \text {,* }}$ \\ 1 Key Laboratory of Bio-Resource and Eco-Environment of Ministry of Education, College of Life Sciences, \\ Sichuan University, Chengdu 610065, China; zjj20045@163.com (J.-J.Z.); guoxinshangan@sohu.com (X.G.); \\ bsyue@scu.edu.cn (B.-S.Y.) \\ 2 Department of Pharmaceutical and Biological Engineering, School of Chemical Engineering, \\ Sichuan University, Chengdu 610065, China; cusack@scu.edu.cn \\ 3 Sichuan Gooddoctor-Panxi Pharmaceutical Co., Ltd., Xichang 615000, China; mxy0701@hys.cn \\ * Correspondence: ljtjf@126.com; Tel.: +86-138-0806-7169
}

Received: 12 December 2017; Accepted: 31 December 2017; Published: 20 January 2018

\begin{abstract}
Ethanol extract (EE) from Periplaneta americana (PA) is the main ingredient of Kangfuxin, which is a popular traditional chinese medicine (TCM) and has long been used for the clinical treatment of burns, wounds and ulcers. We compared the wound-healing activities of three extracts of PA using cutaneous wound-healing in mice as the bioactivity model. These three extracts were EE, total polysaccharide and total protein. We also tracked bioactive fractions in the EE by organic reagent extraction, column chromatography and HPLC. Seven compounds were successfully identified from the water elution fraction of the EE of PA using UPLC-MS. Among these compounds, four compounds (P2, P3, P4, P5(1)) were first reported in PA. Some of these compounds have been previously reported to have various pharmacological activities that could contribute to the high wound-healing activity of PA.
\end{abstract}

Keywords: Periplaneta americana; wound-healing activity; active constituents; bioactivity-guided screening

\section{Introduction}

Wound healing is a complex biological process including three classic stages: inflammation, new tissue formation and remodeling [1]. Any aberrancies at each stage can lead to delayed wound healing. Many synthetic drugs and artificial skins are used for wound healing, but they are expensive and readily cause allergic reactions in sensitive people [2-4]. Thus, there is a real need for an alternative to synthetic wound-healing products. Natural products are the most reliable and successful sources of drug leads. For example, insects have been increasingly used as effective bioactive products and as they are one of the most diverse taxa of living organisms can provide a considerable resource of potential alternatives [5]. Therefore, the potential for insect constituents, such as cockroaches, to enhance natural wound healing is a particularly important research avenue.

Periplaneta americana (PA), the American cockroach, has the largest body size in the family Blattidae. It is also one of the most famous sanitation-related insects with strong vitality and successful reproduction [6]. PA is widely distributed in subtropical and tropical regions across the world [7]. Many previous PA studies have focused on the infestation of human dwellings and the strong ability to transmit pathogenic fungi [8]. However, other physiological and pharmacological studies have demonstrated that PA constituents have favorable tissue-repairing [9], antibacterial [10], antitumor [11] and immunity-enhancing activities [12]. Additionally, this insect has been used in traditional chinese medicine (TCM) as an important biomedical component for the treatment of TCM syndromes such as 
blood stasis, ulcers, burns and wounds for hundreds of years [13]. Consequently, the formulations of many TCM preparations, such as Kangfuxin Liquid, Ganlong Capsule, Xinmailong Injection, and Xiaozheng Yigan Tablet, include ingredients from PA [14]. Among them, Kangfuxin is a liquid preparation that has been used to treat different skin or mucosa injuries in China for more than 40 years [15]. According to the previous component analysis on PA, the reported chemical components of the cockroach mainly include pheromones, amino acids, insect neuropeptides, adipokinetic hormones and dihydroisocoumarins [16]. However, the effective ingredients in PA that can promote the wound-healing process remain largely unknown. The purpose of this study is to investigate related active chemical components that demonstrate efficacy in wound healing. In the present study, we systematically screened the bioactive compounds or fractions with wound-healing activities in PA. The ethanol extract (EE), total polysaccharide and total protein of PA were used to compare their wound-healing activities on the basis of the cutaneous injured mice model. Then, we performed systematic separation and analysis on the EE of PA, including organic reagent extraction, macroporous resin column chromatography, High Performance Liquid Chromatography (HPLC) and Ultra Performance Liquid Chromatography-Mass Spectrometry (UPLC-MS).

\section{Results and Discussion}

\subsection{Comparison of Wound-Healing Activities of Different PA Extracts}

The wound-healing activities of the EE, polysaccharide (TPS) and total protein (TP) of PA are shown in Figure 1a. Compared to other PA extracts, the EE-treated wounds became dry, and scabs began to form on the first day of treatment, likely as a result of the adjacent skin cells interacting with the scab to promote wound healing [17]. The sizes of the wounds decreased significantly at the third and sixth day in the EE-treated group. Scabs of wounds treated with EE started to drop off on the ninth day, and the granulation tissue below the wound grew swiftly and showed a pinkish color. However, pus and blood were observed in the wounds of the TPS and TP group mice even after the sixth day. The results indicated that EE-treated wounds scabbed faster, and had smaller wound sizes and less swelling than those treated with TPS, TP and Jingwanhong (i.e., positive control-PC).

(a)

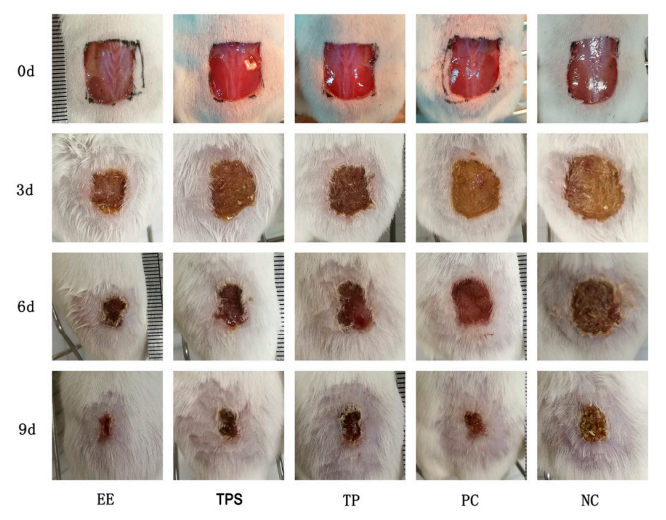

(b)

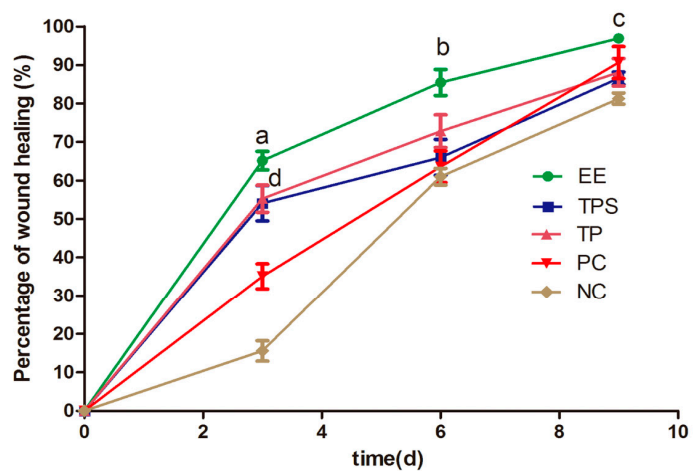

Figure 1. Wound-healing assay of mice treated with ethanol extract (EE), total polysaccharide (TPS), total protein (TP), Jingwanhong (positive control-PC) and pure water (negative control-NC) at 0, 3, 6 and 9 days. (a) Photographs of dorsal excisional skin wounds on different days. Day 0 pictures were taken immediately after wounding; (b) Data are given as mean \pm standard deviation (SD) for three mice in each group. Statistical analysis of wound area used one-way ANOVA followed by Tukey's Honestly Significant Difference (HSD) post hoc test. The characters indicate statistically significant differences $(p<0.05): \mathrm{a}, \mathrm{b}$ and $\mathrm{c}$ : EE compared with other four groups on days 3, 6 and $9 ; \mathrm{d}$ : between TP/TPS and NC on day 3. 
We found that the healing rates of the EE-treated group were the highest at each time interval compared to the TPS, TP and negative control (NC) groups (Figure 1b). Moreover, the healing rate of the EE-treated group significantly increased $(p<0.05)$ to $65 \%$ at the third day, whereas the healing rate was only $16 \%$ in the NC group. We noted that the percentage increases in wound healing slowed at the sixth day and ninth day, which may be related with the formation of scabs. Previous studies have suggested that the scabs could slow the wound-healing process and prevent the migration of epithelium cells on the surface of the wound exudates [18,19]. We also noted that Jingwanhong-treated wounds were slow to heal at day 3 but exhibited a similarly high healing rate (91\%) to the EE-treated group at the ninth day. These results suggested that different wound-healing active compounds might exist in the EE of PA and Jingwanhong. In addition, the TPS- and TP-treated groups exhibited significantly high healing rates only at the third day as compared to both of the control groups $(p<0.05)$.

\subsection{Separation by Organic Reagent Extraction and Macroporous Resin Column Chromatography}

EE was separated by organic reagent extraction to obtain chloroform fraction Fr.A (0.3169 g), ethyl acetate fraction Fr.B (0.5208 g), n-butanol fraction Fr.C (4.3195 g) and water fraction Fr.D $(55.5534 \mathrm{~g})$. The yield rates and characteristics of the four fractions with organic reagent extraction are shown in Table 1. It was found that the yield rates increased with the polarity of the extracted organic reagents, with the lowest yield in the chloroform fraction (Fr.A) and the highest yield in the water fraction (Fr.D) (11\%). The bioactivity analysis also indicated that Fr.D had a better wound-healing activity than the other three fractions. When Fr.D was applied to the cutaneous wounded mice, the wound-healing rate reached $93.6 \%$ at the ninth day, whereas it reached only $9.4 \%$ when Fr.A was applied (Table 1). The results indicated that highly polar constituents predominant in the EE may contribute to the wound-healing activity.

Table 1. The yield, color and healing rate of different solvent extracts (Fr.A: chloroform fraction; Fr.B: ethyl acetate fraction; Fr.C: $n$-butanol fraction; Fr.D: water fraction D) of $500 \mathrm{~mL}$ of Periplaneta Americana (PA) ethanol extract.

\begin{tabular}{cccc}
\hline Extract & Yield (\%) & Color of Concentrate & Healing Rate (Mean \pm SD) $\mathbf{o}^{* *}$ \\
\hline Fr.A & 0.063 & Red brown & $9.4 \pm 1.4$ \\
Fr.B & 0.104 & Red brown & $75.4 \pm 3.7$ \\
Fr.C & 0.864 & Tan & $61.5 \pm 5.6$ \\
Fr.D & 11.111 & Black brown & $93.6 \pm 4.7$ \\
\hline
\end{tabular}

* The yield rate means the proportion of various solvent extracts in the PA powder. ** The healing rate of various extracts on day 9 (pure water-negative control group: $71.3 \pm 3.4 \%$; Jingwanhong-positive control group: $92.3 \pm 5.8 \%)$. SD: standard deviation.

Fr.D (30 g) was then fractionated on macroporous resin column with different gradients of ethanol generating six subfractions of water elution fraction Fr.D1 (23.184 g), 10\% ethanol fraction Fr.D2 (1.604 g), 30\% ethanol fraction Fr.D3 (1.87 g), 50\% ethanol fraction Fr.D4 (0.404 g), 70\% ethanol fraction Fr.D5 (0.02 g) and 90\% ethanol fraction Fr.D6 (0.007 g). The Fr.D1 had the highest yield of 77.3\%, and it showed the highest wound-healing activity compared to the other subfractions. When the bioactivities of Fr.D and Fr.D1 were compared (Table 2), Fr.D1-treated wounds showed a nearly 100\% healing rate at the ninth day, while the healing rate of the Fr.D-treated group was around 92\%. Additionally, Fr.D1-treated mice showed better recovery symptoms, including a light pink dermis without any crusts, prominent wound contraction and re-epithelialization, and active granulation tissue, compared to Fr.D. The results suggested that wound-healing active compounds in EE had been concentrated after column chromatography. 
Table 2. Comparison of water fraction Fr.D and water elution fraction Fr.D1 on day 9 in wound-healing (PC: positive control group; NC: negative control group) activities.

\begin{tabular}{ccc}
\hline Fraction & Color ${ }^{*}$ & Healing Rate (Mean \pm SD) * $^{*}$ \\
\hline Fr.D & Red & $92.3 \pm 2.8$ \\
Fr.D1 & Light pink & $99.5 \pm 0.8$ \\
PC & Light pink & $92.6 \pm 2.3$ \\
NC & Reddish-brown & $71.3 \pm 3.4$ \\
\hline
\end{tabular}

* The color and healing rate of wounds on day 9. SD: standard deviation.

\subsection{UPLC-MS Analysis of Wound-Healing Activity Fraction}

The UPLC chromatogram of Fr.D1 (Figure 2a) illustrated five peaks (P1-P4 and P6), which were in agreement with those shown in the total ion chromatogram (Figure 2b). We noted that three additional peaks (P5, P5(1) and P7) were detected in the total ion chromatogram but were absent in the UPLC chromatogram. This result suggested that P5, P5(1) and P7 were highly likely to be compounds with a weak absorption at $254 \mathrm{~nm}$. In addition to the five distinct peaks, three peaks with low response values at retention times of 2.12, 2.92 and $5.37 \mathrm{~min}$ were detected in UPLC. They could not correspond to those of the total ion current because of improper mass spectrometry conditions and ion mode. The corresponding mass chromatograms of seven compounds are shown in Supplemental Material (Figure S1).

(a)

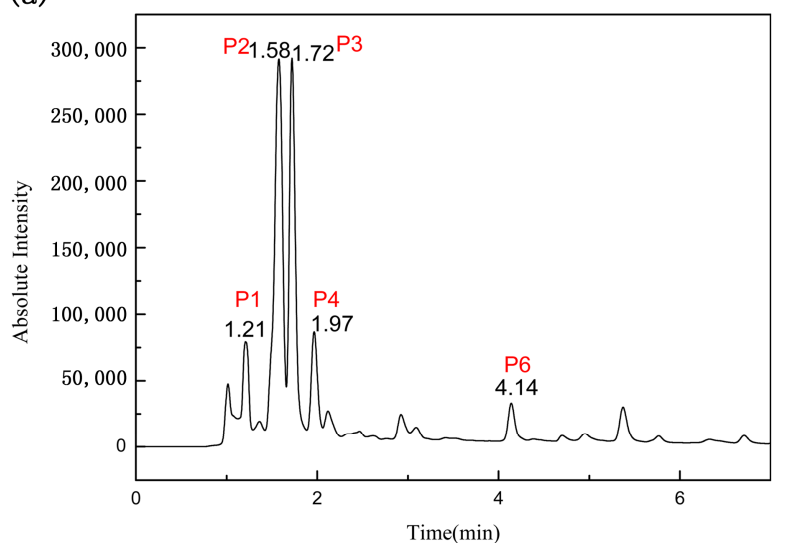

(b)

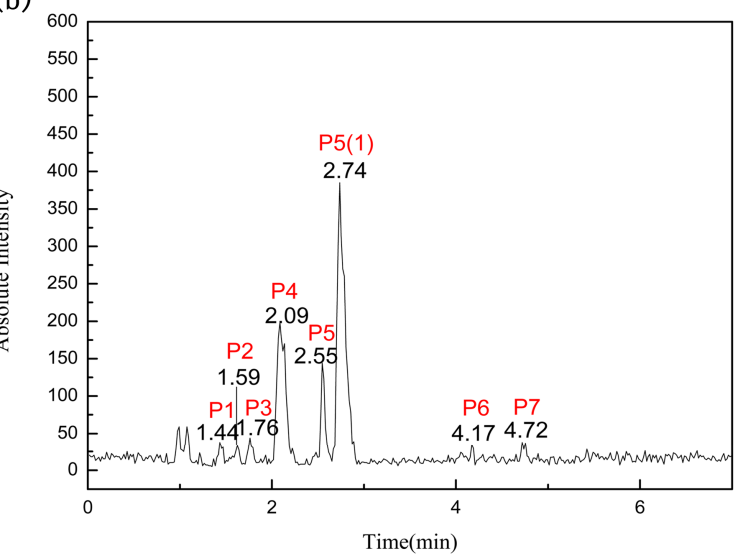

Figure 2. UPLC chromatogram (five peaks) (a) and total ion chromatogram (seven peaks) (b) at $254 \mathrm{~nm}$ (here are shown only intercept chromatograms for the first $7 \mathrm{~min}$ because there was no peak after then).

By integrating UPLC with HR-MS (MS1), we successfully identified seven compounds in the Fr.D1 corresponding to peaks P1-P7. The molecular weight, structure and molecular formula of the compounds were searched for in the SCIfinder database and relevant literature (Table 3). Compounds P5 and P5(1) in Figure 2b were highly likely to be isomers according to the mass spectrometry data. Here we only identified compound P5(1) with a high content. Three of the seven compounds have been previously reported (compound 1: cyclo-(L-Val-L-Pro); compound 6: 7-hydroxycotadecanoic acid; compound 7: (S)-2,3-dihydroxypropyl hexadecanoic acid ester). Whereas the other four compounds (compound 2: 2-(4'-methyl-3'-pentene)-6-hydroxymethyl-10-methyl-12-hydroxyl-(2,6,10)-triendodecanic acid; compound 3: arbutin; compound 4: 4-benzyloxy-3-methoxybenzoic acid; compound 5(1): (E)-3-hexenyl- $\beta$-D-glucopyranoside) were first reported in PA. 
Table 3. Compounds identified from the water elution fraction (Fr.D1) of Periplaneta Americana (M.F.: molecular formula).

\begin{tabular}{|c|c|c|c|c|c|c|}
\hline No. & Ion Peak & $m / z$ & Compound & M.F. & Structure & Reference \\
\hline P1 & {$[\mathrm{M}+\mathrm{K}]^{+}$} & 235.1451 & Cyclo-(L-Val-L-Pro) & $\mathrm{C}_{10} \mathrm{H}_{16} \mathrm{~N}_{2} \mathrm{O}_{2}$ & & [20] \\
\hline P2 & {$[\mathrm{M}+\mathrm{H}]^{+}$} & 337.0458 & $\begin{array}{l}\text { 2-(4'-Methyl-3'-pentene)-6-hydroxymethyl-10-methyl-12 } \\
\text {-hydroxyl-(2,6,10)-triendodecanic acid }\end{array}$ & $\mathrm{C}_{20} \mathrm{H}_{32} \mathrm{O}_{4}$ & & [21] \\
\hline P3 & {$[\mathrm{M}+\mathrm{H}]^{+}$} & 273.0745 & Arbutin & $\mathrm{C}_{12} \mathrm{H}_{16} \mathrm{O}_{7}$ & & [22] \\
\hline P4 & {$[\mathrm{M}+\mathrm{H}]^{+}$} & 259.0785 & 4-Benzyloxy-3-methoxybenzoic acid & $\mathrm{C}_{15} \mathrm{H}_{14} \mathrm{O}_{4}$ & & [23] \\
\hline P5(1) & {$[\mathrm{M}+\mathrm{H}]^{+}$} & 263.1840 & (E)-3-Hexenyl- $\beta$-D-glucopyranoside & $\mathrm{C}_{12} \mathrm{H}_{22} \mathrm{O}_{6}$ & & [24] \\
\hline P6 & {$[\mathrm{M}+\mathrm{K}]^{+}$} & 339.0539 & 7-Hydroxycotadeca-noic acid & $\mathrm{C}_{18} \mathrm{H}_{36} \mathrm{O}_{3}$ & & [25] \\
\hline P7 & {$[\mathrm{M}+\mathrm{Na}]^{+}$} & 331.1576 & (S)-2,3-Dihydroxypropyl hexadecanoic acid ester & $\mathrm{C}_{19} \mathrm{H}_{38} \mathrm{O}_{4}$ & & [7] \\
\hline
\end{tabular}


Compounds 3 and 5(1) are glycosides that have not been previously reported in PA. Compound 3 (arbutin) displayed a $[\mathrm{M}+\mathrm{H}]^{+}$ion at $m / z 273.0745$ and a molecular formula $\mathrm{C}_{12} \mathrm{H}_{16} \mathrm{O}_{7}$ (molecular weight: $272.0745 \mathrm{Da}$ ). The peak area of compound 3 was high in the UPLC chromatogram compared to other compounds, which indicated a high content of arbutin in Fr.D1. As a naturally occurring glycoside, arbutin is known for various pharmacological activities, particularly the anti-inflammation and anti-ulcer activities [26]. Arbutin has been found in several plants, such as Vaccinium vitis idaeae [27], Pyrus bretschneideri [28] and Onobrychis viciaefolia Scop [22]. However, it has not been found in PA or other insects. Three different periplanosides have been previously isolated from PA that share similar structures with arbutin [16]. One of the three periplanosides stimulates collagen production that is closely related with skin and ulcer repair [16]. Considering the similar structure to other periplanosides with high pharmacological activities and the high content in Fr.D1, arbutin was probably an important component contributing to the observed wound-healing activity. Compound 5(1) showed a $[\mathrm{M}+\mathrm{H}]^{+}$ion at $m / z 263.1840$ and the molecular formula $\mathrm{C}_{12} \mathrm{H}_{22} \mathrm{O}_{6}$. It was originally extracted from Hylomecon vernalis Maxim with little cytotoxicity against some human tumor cell lines [24] and was first found in PA.

Compounds 2 (a diterpenoid) and 4 (a phenolic acid) were identified from the Fr.D1 of PA for the first time. Compound 2 showed a $[\mathrm{M}+\mathrm{H}]^{+}$ion at $m / z 337.0458$, and the molecular formula was $\mathrm{C}_{20} \mathrm{H}_{32} \mathrm{O}_{4}$. It was originally isolated from the aqueous extract of Smallanthus sonchifolius, and the crude extract of this plant has been found to have antidiabetic activity [21]. Research on diterpenoids extracted from various plants has confirmed several biological activities, for example, anti-inflammation [29], antimicrobial [30], antitumor [31] and antioxidant [32] activities. Compound $4\left(\mathrm{C}_{15} \mathrm{H}_{14} \mathrm{O}_{4}\right)$ had an elemental composition of the $[\mathrm{M}+\mathrm{H}]^{+}$cation at $m / z 259.0785$ and was reported with high antioxidant activities [23]. Antioxidants can eliminate free radicals that are involved in inflammatory and cardiovascular diseases [33].

Compound 1 cyclo-(L-Val-L-Pro) is a cyclic peptide, which was 1 of 10 reported cyclic peptides initially separated from the ethyl acetate fraction of the PA EE [20]. Previous research has suggested that many natural cyclic peptides have more stable structures than linear peptides and have diverse biological activities such as antitumor, immune-regulatory and antimicrobial activities [34]. Therefore, this compound may have played a role in the process of the cutaneous wound repair of the studied mice.

Compounds 6 and 7 are long-chain fatty acids that were previously isolated from the ethyl acetate extract and the chloroform extract of PA $[7,25]$, respectively. Many types of fatty acids have been identified in PA, including long-chain polyunsaturated fatty acids, benzoic acid and squalene, some of which showed biological activities, such as anti-inflammation, antibacterial and antitumor activities [35-37].

Considering that wound healing is a dynamic and extremely complicated process [1], synergistic effects from different bioactive compounds likely contribute to the high wound-healing activity of Fr.D1. Further studies are expected to elucidate the wound-healing compounds in PA.

\section{Materials and Methods}

\subsection{Reagents and Experimental Animals}

Chloroform, ether, ethanol, ethyl acetate, and $n$-butanol for separation were of analytical grade. Methanol and acetic acid for UPLC were of chromatographic grade. D101 macroporous resin was purchased from the Lingyun Instrumental Factory (Chengdu, China). Jingwanhong (No. 211991), a traditional Chinese medicine for burns, scalds and wound healing [38] and produced from the Pharmaceutical Industry Limited Company (Tianjin, China), was used as the PC. Male Specific pathogen Free (SPF) Kunming mice weighing 18-22 grams were purchased from the Chengdu Dashuo Experimental Animal Limited Company (Chengdu, China). 


\subsection{Preparation of Three PA Extracts}

PA was obtained from the Good Agriculture Practice (GAP) breeding base, Sichuan, China. The powdered dried PA (500 g) was extracted with $90 \%$ ethanol (3 L) twice at $80{ }^{\circ} \mathrm{C}$. After solvent evaporation, the EE was recovered and the yield rate in the PA powder was $21 \%$. The EE was dissolved in distilled water $(100 \mathrm{mg} / \mathrm{mL})$ for bioactivity assays. TPS and TP of PA were both supplied by the molecular biology laboratory of the College of Life Science, Sichuan University. TPS was extracted from PA powder using $0.02 \mathrm{~mol} / \mathrm{L}$ of $\mathrm{NaOH}\left(70{ }^{\circ} \mathrm{C}, 2 \mathrm{~h}\right)$ with a yield rate of $17.0 \%$ in PA powder, whereas TP was extracted by tris- $\mathrm{HCl}\left(35^{\circ} \mathrm{C}, 6 \mathrm{~h}, \mathrm{pH} 6.0\right)$ with a yield rate of $16.2 \%$. TPS and TP were dissolved in distilled water at concentrations of $100 \mathrm{mg} / \mathrm{mL}$ for the bioactivity assays. EE showed the highest activity among the three PA extracts and was then chosen for the following isolation.

\subsection{Organic Reagent Extraction and Macroporous Resin Column Chromatography Isolation}

The organic reagent extraction for EE was based on the method of Yuanhui Li [39]. EE (500 mL) was sequentially extracted three times $(500 \mathrm{~mL} \times 3)$ with chloroform, ethyl acetate and $n$-butanol. All layers were combined and concentrated under vacuum to obtain the chloroform fraction (Fr.A), ethyl acetate fraction (Fr.B), n-butanol fraction (Fr.C) and water fraction D (Fr.D). All of the samples were maintained at $-20{ }^{\circ} \mathrm{C}$ until use. Among these fractions, Fr.D showed the highest bioactivity and was subsequently used for further macroporous resin column chromatography.

D101-type resin (300 g) was weighed and soaked in ethanol for $24 \mathrm{~h}$, and then a resin column $(4 \mathrm{~cm} \times 50 \mathrm{~cm})$ was packed by a wet method [40]. Subsequently, absolute ethanol and distilled water were used to wash the resin in turn until there was nearly no turbidity in elution. Then $30 \mathrm{~g}$ of Fr.D was loaded after being dissolved in distilled water. After the sample was completely absorbed, the resin was successively eluted with an increasing gradient ethanol at a flow rate of $3 \mathrm{~mL} / \mathrm{min}$. Six fractions were yielded, Fr.D1, Fr.D2, Fr.D3, Fr.D4, Fr.D5 and Fr.D6. All fractions were condensed and weighed. All samples were maintained at $-20^{\circ} \mathrm{C}$ for use. The Fr.D1 possessed a better activity than Fr.D and was followed by analytical HPLC. The scheme of extraction and isolation procedure is shown in Figure 3.

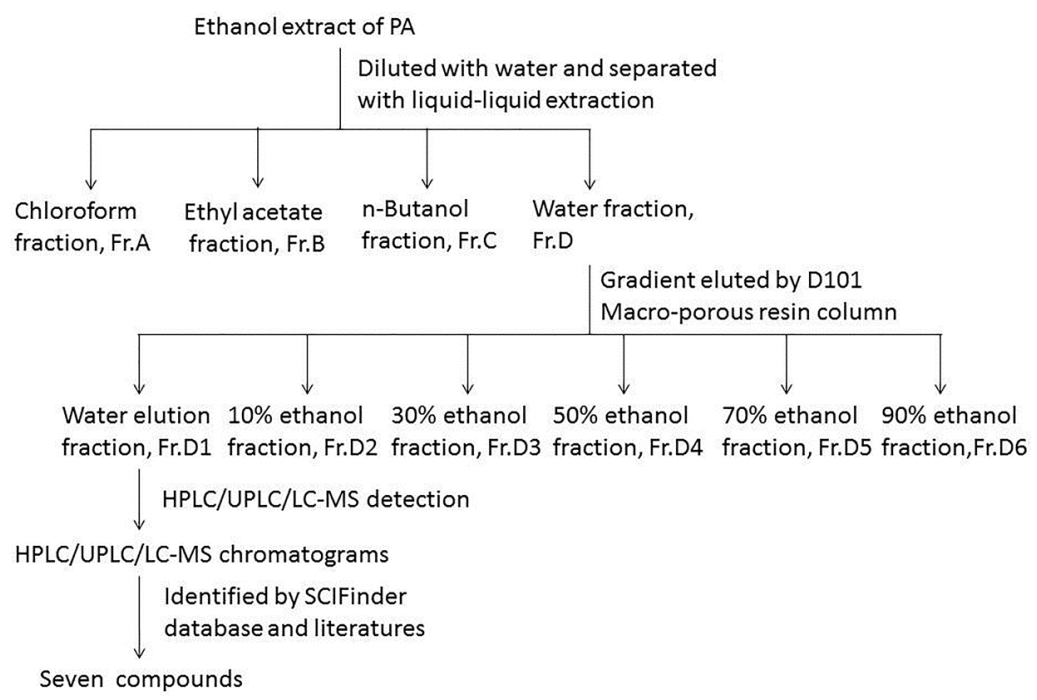

Figure 3. Separation and analysis procedure of ethanol extract from Periplaneta americana (PA).

\subsection{UPLC-MS Identification and Analysis}

Chromatographic separation was performed on a Waters Acquity UPLC system equipped with a C18 column $(2.1 \times 100 \mathrm{~mm}, 1.8 \mu \mathrm{m})$. The mobile phase consisted of solvents $\mathrm{A}(0.1 \%$ formic acid in water) and B (methanol). The injection volume was $5 \mu \mathrm{L}$. A flow rate of $0.2 \mathrm{~mL} / \mathrm{min}$ for a total run time of $15 \mathrm{~min}$ with a linear gradient was used as follows: 0-2 $\mathrm{min}, 3-5 \% \mathrm{~B} ; 2-5 \mathrm{~min}, 5-10 \% \mathrm{~B} ; 5-10 \mathrm{~min}$, 
10-20\% B; 10-15 min, 20-30\% B. The UPLC-MS analysis was carried out using a Q-TOF Premier system with an electrospray ionization (ESI) source in the positive ion mode (Quattro Premier XE Mass Spectrometer, Waters, USA). The main parameters of UPLC-MS were as follows: capillary voltage at $2.8 \mathrm{eV}$, extraction cone at $3.5 \mathrm{~V}$, cone gas flow at $25 \mathrm{~L} / \mathrm{h}$, source temperature at $90{ }^{\circ} \mathrm{C}$, collision energy at $5 \mathrm{eV}$, sampling cone at $40 \mathrm{~V}$, desolvation temperature at $200{ }^{\circ} \mathrm{C}$, and desolvation gas flow at $300 \mathrm{~L} / \mathrm{h}$. The chromatograms and mass spectral data were compared with that of the reference data, and the molecular weight, formula and structure of the compounds identified were determined through the SCIfinder database.

\subsection{Assay of Wound-Healing Activity}

The wound-healing activities of EE, TPS and TP of the PA extracts were compared. Next, EE was further divided into Fr.A, Fr.B, Fr.C and Fr.D, and the wound-healing activities of each fraction were measured. The activities of Fr.D and Fr.D1 isolated from Fr.D were also investigated.

\subsubsection{Animal Modeling of Cutaneous Excision Wound and Administration}

All male SPF mice were housed at room temperature and fed with water and food freely for one week to acclimatize to laboratory conditions before the experiment. All experimental procedures were in accordance with the "Guidelines for the Care and Use of Animals in Research" of the Institute of Zoology, Chinese Academy of Sciences. The mice were randomly divided into experimental groups, a PC group and a NC group, with three animals in each group.

All of the mice were inhibited from drinking water the night before the operation. The hair on the dorsal side of the mice was removed with $8 \%$ sodium sulfide, and then the mice were put back in cages after cleaning the exposed skin area with warm water. On the day of the surgery, the mice were anesthetized by an intraperitoneal injection of $10 \%$ chloral hydrate $(0.003 \mathrm{~mL} / \mathrm{g})$, and a $1.4 \mathrm{~cm} \times 1.4 \mathrm{~cm}$ symmetrical square was outlined along the dorsal middle line. The skin was sterilized using $75 \%$ alcohol, and a wound was created by excision of the superficial fascia according to the outlined square [41,42]. The mice were then housed individually in cages.

The mice of the experimental groups and NC group were treated with $30 \mu \mathrm{L}$ of extracts and water by dripping slowly after $2 \mathrm{~h}$ of modeling, respectively, while Jingwanhong ointment was evenly applied onto the wound area with medical cotton stickers of the PC group mice. The wounds were left open after administering either the treatment or control, and the mice were put back into clean cages. The administration for each group was conducted once a day for 9 days. In the process of modeling and administration, the mice all showed a good mental state and activity.

\subsubsection{Evaluation of Wound-Healing Activity and Statistical Analysis}

The cutaneous wounds of mice were photographed, and the wound areas were measured by tracing the margins after the injury (day 0), on the third, sixth and ninth days. The percentage of wound healing was calculated with the following formula [41]:

Percentage of wound healing $(\%)=($ original area - unhealed area $) /$ original area $\times 100$

The wound-healing percentage was analyzed by one-way ANOVA followed by Tukey's HSD post hoc test using statistical software package (SPSS) and GraphPad Prism software (version 5.0, GraphPad Software Inc., San Diego, CA, USA). All results are displayed as mean \pm standard deviation (SD), and $p<0.05$ was considered as statistically significant.

\section{Conclusions}

Through the combination of traditional separation strategies with bioactivity tracking, we successfully found that the water elution fraction (Fr.D1) of EE of PA had the highest healing activity. Seven compounds (including cyclopeptide, diterpenoid, phenolic acid, fatty acids and glycosides) were 
identified from this fraction with the UPLC-MS method, among which the diterpenoid, one phenolic acid and the two glycosides were first reported in PA. Most of the identified compounds have been reported to have antibacterium, anti-inflammation or enhancing immunity activities that were highly likely to be associated with wound repair for mice. The study is expected to lay the foundation for further research and utilization of PA.

Supplementary Materials: The supplementary materials are available online.

Acknowledgments: This work was supported by the Sichuan Gooddoctor-Panxi Pharmaceutical Company Limited and Key Laboratory of Bio-Resource and Eco-Environment of Ministry of Education, College of Life Sciences, Sichuan University.

Author Contributions: Juan-Juan Zhu, Shun Yao and Jing Li designed the experiments; Juan-Juan Zhu performed the experiments; Juan-Juan Zhu and Xin Guo analyzed the data; Xiu-Ying Ma provided experimental materials; Juan-Juan Zhu wrote the paper. All authors read and approved the final manuscript.

Conflicts of Interest: The authors declare no conflict of interest.

\section{References}

1. Gurtner, G.C.; Werner, S.; Barrandon, Y.; Longaker, M.T. Wound repair and regeneration. Nature 2008, 453, 314-321. [CrossRef] [PubMed]

2. Boateng, J.S.; Matthews, K.H.; Stevens, H.N.; Eccleston, G.M. Wound healing dressings and drug delivery systems: A review. J. Pharm. Sci. 2008, 97, 2892-2923. [CrossRef] [PubMed]

3. Macdonald, J.; Asiedu, K. WAWLC: World a alliance for wound and lymphedema care. Wounds 2010, 22, 55-59. [PubMed]

4. Oprea, A.M.; Ciolacu, D.; Neamtu, A.; Mungiu, O.C.; Stoica, B.; Vasile, C. Cellulose/chondroitin sulfate hydrogels: Synthesis, drug loading/release properties and biocompatibility. Cell. Chem. Technol. 2010, 44, 369-378.

5. Suh, H.J.; Kim, S.R.; Lee, K.S.; Park, S.; Kang, S.C. Antioxidant activity of various solvent extracts from Allomyrina dichotoma (Arthropoda: Insecta) larvae. J. Photochem. Photobiol. B 2010, 99, 67-73. [CrossRef] [PubMed]

6. Zou, J.B.; Sang, W.T.; Wang, F.; Yang, S.; Zhang, T.; Zeng, N. Protective effect of Periplaneta americana extract on mice with ethanol-induced acute gastric ulcer. Chin. Tradit. Pat. Med. 2016, 38, 2325-2331.

7. Jiang, W.X.; Luo, S.L.; Wang, Y.; Wang, L.; Zhang, X.; Ye, W.C. Chemical constituents from Periplaneta americana. J. Jinan Univ. 2015, 36, 294-301.

8. Nasirian, H. Infestation of cockroaches (Insecta: Blattaria) in the human dwelling environments: A systematic review and meta-analysis. Acta Trop. 2017, 167, 86-98. [CrossRef] [PubMed]

9. Xie, B.; Shu, Y.; Liang, Z.; Chen, J.H.; Cheng, Y.N.; Zhang, X.N. Effect of Periplaneta americana extraction on post-injury healing of rat bladder mucosa. Zhejiang J. Integr. Tradit. Chin. West. Med. 2012, 22, 689-691.

10. Wang, K.; Feng, Y.; Sun, L.; He, Z.; Chen, Z.Y. Isolation of ethyl acetate extract from Periplaneta americana and its antimicrobial activity. For. Res. 2013, 26, 163-166.

11. Wang, J. Effect of Periplaneta americana extract on H125 lung cancer cells. Chin. J. Public Health 2014, 30, 1400-1402.

12. Zhou, Q.; Li, Z.R.; Liu, J.; Lin, Q.; Wang, C.K.; Jiang, X. Effect of Periplaneta americana meal on immunity and antioxidation of broliers. J. Fujian Agric. For. Univ. 2009, 38, 175-180.

13. Alves, R.R.; Alves, H.N. The faunal drugstore: Animal-based remedies used in traditional medicines in Latin America. J. Ethnobiol. Ethnomed. 2011, 7, 9. [CrossRef] [PubMed]

14. Luo, T.S.; Gao, M.T.; Ma, F.F.; Liu, G.M.; Zhang, C.G. Research advances in pharmacological action and clinical application of Periplaneta americana. J. Anhui Agric. Sci. 2012, 40, 5933-5935.

15. Zhang, J.; Meng, L.H.; Shan, S.J.; Zhang, L.T. Effect of Kangfuxin liquid on subcrustal wound healing of rat. J. Tianjin Med. Univ. 2014, 20, 192-195. 
16. Yang, Y.X.; Luo, Q.; Hou, B.; Yan, Y.M.; Wang, Y.H.; Tang, J.J.; Dong, X.P.; Ma, X.Y.; Yang, T.H.; Zuo, Z.L.; et al. Periplanosides A-C: New insect-derived dihydroisocoumarin glucosides from Periplaneta americana stimulating collagen production in human dermal fibroblasts. J. Asian Nat. Prod. Res. 2015, 17, 988-995. [CrossRef] [PubMed]

17. Usui, M.L.; Underwood, R.A.; Fleckman, P.; Olerud, J.E. Parakeratotic corneocytes play a unique role in human skin wound healing. J. Investig. Dermatol. 2013, 133, 856-858. [CrossRef] [PubMed]

18. Drew, A.F.; Liu, H.; Davidson, J.M.; Daugherty, C.C.; Degen, J.L. Wound-healing defects in mice lacking fibrinogen. Blood 2001, 97, 3691-3698. [CrossRef] [PubMed]

19. Guo, S.; Dipietro, L.A. Factors affecting wound healing. J. Dent. Res. 2010, 89, 219-229. [CrossRef] [PubMed]

20. Li, Y.; Wang, F.; Zhang, P.; Yang, M. Chemical Constituents from Periplaneta americana. Chin. Med. Mater. 2015, 38, 2038-2041.

21. Tian, F. Chemical constituents of Artemisia borealis and antidiabetic components of the leaves of Smallanthus sonchifolius. Master's Thesis, Liaoning University of Traditional Chinese Medicine, Liaoning, China, 2007.

22. Regos, I.; Urbanella, A.; Treutter, D. Identification and quantification of phenolic compounds from the forage legume sainfoin (Onobrychis viciifolia). J. Agric. Food Chem. 2009, 57, 5843-5852. [CrossRef] [PubMed]

23. Catel, Y.; Aladedunye, F.; Przybylski, R. Synthesis, radical scavenging activity, protection during storage, and frying by novel antioxidants. J. Agric. Food Chem. 2010, 58, 11081-11089. [CrossRef] [PubMed]

24. Lee, S.Y.; Kim, K.H.; Lee, I.K.; Lee, K.H.; Choi, S.U.; Lee, K.R. A new flavonol glycoside from Hylomecon vernalis. Arch. Pharm. Res. 2012, 35, 415-421. [CrossRef] [PubMed]

25. Tulloch, A.P. Carbon-13 NMR spectra of all the isomeric methyl hydroxy- and acetoxyoctadecanoates. Determination of chemical shifts by deuterium isotope effects. Magn. Reson. Chem. 2010, 11, 109-115.

26. Tang, J.Y.; Peng, F. Research progress of pharmacological effects and ways of obtaining resources of arbutin. Pharm. Today 2015, 25, 673-677.

27. Pyka, A.; Bober, K.A. Densitometric determination of arbutin in cowberry leaves (Vaccinium vitis idaeae). Acta Pol. Pharm. 2007, 64, 395-400. [PubMed]

28. Tong, C.; Nakamura, K.; Ma, L.; Li, J.Z.; Hiroshi, K. Analyses of arbutin and chlorogenic acid, the major phenolic constituents in oriental pear. J. Agric. Food Chem. 2005, 53, 3882-3887.

29. Feng, Z.L.; Li, D.; Liu, Q.Y.; Liu, J.X.; Huang, L.; Zhang, Q.W.; Wang, Y.T.; Lin, L.G. Anti-inflammatory abietane diterpenoids from the seeds of Podocarpus nagi. Phytochem. Lett. 2017, 21, 260-263. [CrossRef]

30. Amugune, B.; Milugo, T.K.; Heydenreich, M.; Omosa, L.K.; Ndunda, B.; Yenesew, A.; Midiwo, J.O. Antimicrobial flavonoids and diterpenoids from Dodonaea angustifolia. S. Afr. J. Bot. 2014, 91, 58-62.

31. Zhao, D.K.; Shi, X.Q.; Zhang, L.M.; Yang, D.Q.; Guo, H.C.; Chen, Y.P.; Shen, Y. Four new diterpenoid alkaloids with antitumor effect from Aconitum nagarum var. heterotrichum. Chin. Chem. Lett. 2016, 28, 358-361. [CrossRef]

32. Bajpai, V.K.; Baek, K.H.; Kang, S.C. Antioxidant and free radical scavenging activities of taxoquinone, a diterpenoid isolated from Metasequoia glyptostroboides. S. Afr. J. Bot. 2017, 111, 93-98. [CrossRef]

33. Sachidanandam, K.; Fagan, S.C.; Ergul, A. Oxidative stress and cardiovascular disease: Antioxidants and unresolved issues. Cardiovasc. Ther. 2005, 23, 115-132. [CrossRef]

34. Xu, W.Y.; Zhao, S.M.; Zeng, G.Z.; He, W.J.; Xu, H.M.; Tan, N.H. Progress in the study of some important natural bioactive cyclopeptides. Acta Pharmacol. Sin. 2012, 47, 271-279.

35. Yu, X.B.; Li, J.; Huang, X.Z.; Shen, Y.H. Functional properties and future trends in biosynthesis of longchain polyunsaturated fatty acids. Sci. Seric. 2014, 40, 153-160.

36. Zhao, Z.D.; Sun, Z. Research progress on natural resources and application of the bioactive substance-squalene. Inst. Chem. Ind. For. Prod. 2004, 24, 107-112.

37. Cao, H.; Wang, M.W.; Xue, S.Y.; Wei, X.L. The mechanism of triolein and its effects on mucosal necrosis. J. Shenyang Pharm. Univ. 2000, 17, 367-370.

38. Sun, W. Observe and nurse regeneration and repair of skin coated Jingwanhong ointment. Hebei Med. 2006, 12, 1015-1017.

39. Li, Y.H.; Li, X.; Feng, J.A.; Wu, Y.X.; Wu, Y. Research on in vitro antimicrobial activity of different extracts from Periplaneta americana L. Pharm. Clin. Chin. Mater. Med. 2014, 5, 27-29.

40. Wang, Y.; Su, W.; Liang, S. Separation of aqueous extract from Hypericum japonicum Thunb using different types of macroporous resin. Chin. Med. Mat. 2007, 30, 1537-1539. 
41. Yariswamy, M.; Shivaprasad, H.V.; Joshi, V.; Nanjaraj Urs, A.N.; Nataraju, A.; Vishwanath, B.S. Topical application of serine proteases from Wrightia tinctoria $\mathrm{R}$. Br. (Apocyanaceae) latex augments healing of experimentally induced excision wound in mice. J. Ethnopharmacol. 2013, 149, 377-383. [PubMed]

42. Muthukumar, T.; Anbarasu, K.; Prakash, D.; Sastry, T.P. Effect of growth factors and pro-inflammatory cytokines by the collagen biocomposite dressing material containing Macrotyloma uniflorum plant extract-In vivo wound healing. Colloids Surf. B Biointerfaces 2014, 121, 178-188. [CrossRef] [PubMed]

Sample Availability: Samples of the used insect materials are available from the authors.

(C) 2018 by the authors. Licensee MDPI, Basel, Switzerland. This article is an open access article distributed under the terms and conditions of the Creative Commons Attribution (CC BY) license (http://creativecommons.org/licenses/by/4.0/). 